\title{
Balloon Dilatation of Benign Rectal Anastomotic Strictures - A Review
}

\author{
J. Ragg ${ }^{\mathrm{a}} \quad$ V. Garimella ${ }^{\mathrm{a}} \quad$ J. Cast ${ }^{\mathrm{b}} \quad$ I.A. Hunter ${ }^{\mathrm{a}} \quad$ J.E. Hartley ${ }^{\mathrm{a}}$

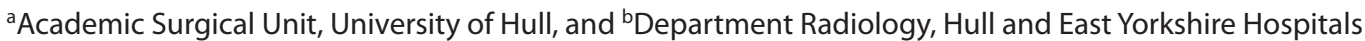 \\ NHS Trust, Cottingham, East Yorkshire, UK
}

\section{Key Words}

Balloon dilatation $\cdot$ Rectal anastomotic strictures $\cdot$ Rectal

stricture symptoms $\cdot$ Stricture dilatation

\begin{abstract}
Background: The occurrence of anastomotic stricture at the level of the rectum gives rise to three broad therapeutic options, namely major pelvic and abdominal revisional surgery, faecal diversion (stoma), or local revision by transanal approaches (including endoscopic and fluoroscopic). This article updates the current evidence and focuses on the results of the balloon dilatation technique. Methods: A Medline search was carried out using the search terms (dilatation OR dilatation) AND (stricture OR strictures OR stenosis OR stenotic) AND (rectum OR rectal). In an effort to lessen publication bias, articles included at least 10 patients who were consecutively referred for treatment. Results/Conclusion: This review would suggest that probably relatively short strictures have been chosen for balloon dilatation and that the results have had a very low major morbidity $(0.45 \%)$ and mortality $(0 \%)$ rate.

Copyright $\odot 2012$ S. Karger AG, Basel
\end{abstract}

\section{Introduction}

The incidence of rectal anastomotic strictures has increased with the widespread use of circular staplers [1,
2]. Therapeutic options for these strictures include major pelvic and abdominal revisional surgery, faecal diversion (stoma) or local revision by transanal approaches (including endoscopic and fluoroscopic approaches).

Garcea et al. [3], in a review article entitled 'Management of benign rectal strictures' (2003), identified just 27 cases of open pelvic revisional surgery to review. Faecal diversion (stoma) treatments were not mentioned. The remaining cases identified to review were transanal approaches to deliver a variety of therapies. The largest group was dilatation only $(\mathrm{n}=136)$, which was predominantly by the balloon technique. Other therapies included strictureplasty ( $n=29$, stapler, hand-sewn methods), electrocautery ( $n=92$, e.g. electrocautery, laser, urologic resectoscope, transanal endoscopic microsurgery technique, and others) and mechanical stenting. This article is to update the evidence on this review article and to focus in more detail on the results of the balloon dilatation technique.

\section{Methods}

Articles that were included were those describing only strictures known to be benign at the time of dilatation, articles describing the technique of balloon dilatation as the main treatment and those which, in an effort to lessen publication bias, included at least 10 patients that were consecutively referred for treatment.

\section{KARGER}

Fax +41613061234

E-Mail karger@karger.ch

www.karger.com
(C) 2012 S. Karger AG, Basel

0253-4886/12/0294-0287\$38.00/0

Accessible online at: www.karger.com/dsu
Mr. J.E. Hartley

Academic Surgical Unit, Castle Hill Hospital

Castle Road, Cottingham HU16 5JQ (UK)

Tel. +44 1482622393

E-Mail J.E.Hartley@hull.ac.uk 
A Medline search was carried out, using the search term (dilatation or dilatations) AND (stricture or strictures or stenosis or stenotic) AND (rectum or rectal). Bibliographies of the identified articles were reviewed. Hyperlinks to suggested related articles that were found when the search was run on the PubMed Central (PMC) United States of America National Institutes of Health search resource were also reviewed to identify suitable publications.

\section{Results}

A total of 12 articles were identified from the literature search [3-16]. In addition, unpublished series from our institution were also included. There were 405 patients having 850 procedures (mean 2.1 procedures per patient). Cumulative recruitment time was 94 years. The mean number of patients recruited per year was thus 4.30, and the mean number of procedures per year was thus 9.04. Only one study gave an overall incidence of stricture after rectal surgery of $7.34 \%$ (26/422) [15]. A second study looked specifically at patients who underwent laparoscopic sigmoid colectomy and found the incidence to be $17.6 \%(12 / 68)$ [4]. Hypothetically, if there were 50-200 anastomoses at the level of rectum performed per year within the referring populations of the institutions associated with the series presented in this review, then $2.1-8.6 \%$ of patients would have been referred for dilatation $(405 /(94 \times 50$ or 200$) \times 100)$.

\section{Symptoms and Signs}

Symptoms most commonly associated with rectal strictures were increasing constipation and large bowel obstruction. Other symptoms included string like stools and spurious diarrhoea. Some patients with/without symptoms were identified on digital rectal examination in outpatient clinics. Patients with diverting stoma were identified to have stricture on routine endoscopy before reversal of stoma. Endoscopically, an anastomosis was deemed to be strictured if it did not permit the end of the scope through it.

\section{The Stricture}

The level of stricture was predominantly in the rectum (92\%) although some cases were reported in the colon and anal canal (table 1). The primary cause of stricture was anastomotic (96\%) with some cases contributed by inflammatory bowel disease and radiotherapy (table 1). The underlying pathology for colonic/rectal resection was carcinoma (76\%), diverticular disease (14\%) and inflammatory bowel disease (4\%) (table 1). Faecal diversion
Table 1. Pathological characteristics of patients who underwent dilatation of strictures

\begin{tabular}{lrc}
\hline & Patients, $\mathrm{n}$ & Percentage \\
\hline Level of stricture & & \\
$\quad$ Anal & 7 & 2 \\
Rectum & 373 & 92 \\
Colon & 25 & 6 \\
Cause of stricture & & \\
$\quad$ Anastomotic & 389 & 96 \\
Crohn's and ulcerative colitis & 6 & 2 \\
Radiation & 5 & 1 \\
Ischaemic & 2 & 0.5 \\
$\quad$ Anal scarring & 3 & 0.5 \\
Underlying pathology & & \\
Carcinoma & 308 & 76 \\
Diverticular disease & $56=+$ & 14 \\
Crohn's and ulcerative colitis & 15 & 4 \\
Radiotherapy & 5 & 1 \\
Adenoma & 2 & 0.5 \\
Ischaemic & 2 & 0.5 \\
\hline
\end{tabular}

from the anastomoses was present in 100/405 (24.6\%) patients at the time of stricture dilatation. The mean duration between operation and first dilatation, weighted for number of patients in each study that gave this information $[4,7,10,11]$, was 9.91 (SD 4.27) months. The mean diameter of stricture, weighted for number of patients in each study that gave this information, was mean 8.62 (SD 2.99) $\mathrm{mm}[2,6,8]$ and the length was mean 14.03 (SD 2.12) $\mathrm{mm}[8,11]$.

\section{Procedures}

The most common procedure used was endoscopic dilatation. Di et al. [8] described the technique of fluoroscopic double balloon dilatation. This involved insertion of a double balloon catheter under fluoroscopic guidance and dilating the two balloons (outer $20 \mathrm{~mm}$ and inner 10, 15 , or $20 \mathrm{~mm}$ diameter). Suchan et al. [15] used either fluoroscopic or endoscopic dilatation of stricture in $82 \%$ (78/94) patients. Of these, 30 patients also underwent laser/argon incision if the diameter of the stricture was $<7 \mathrm{~mm}$ in order to facilitate insertion of the hydrostatic balloon catheter. The rest of the patients underwent laser incision only $(12 / 94,12.7 \%)$ or bougienage $(4 / 94,4 \%)$ as treatment of stricture. Di Giorgio et al. [6] compared endoscopic with fluoroscopic dilatation in a prospective comparative study and Pietropaolo et al. [14] compared endoscopic dilatation with bougienage (table 2). 
Table 2. Number of procedures and success rate for stricture dilatation in individual studies

\begin{tabular}{lllll}
\hline Study (first author) & $\begin{array}{l}\text { Patients } \\
\mathrm{n}\end{array}$ & Type of dilatation & Success (\%) & $\begin{array}{l}\text { Average } \\
\text { number of } \\
\text { dilatations }\end{array}$ \\
\hline Araujo, 2008 [5] & 24 & endoscopic & $22(92)$ & 2.3 \\
Di Giorgio, 2004 [6] & 30 & endoscopic & $30(100)$ & 2.6 \\
Venkatesh, 1992 [12] & 17 & endoscopic & $16(96)$ & - \\
Johansson, 1996 [9] & 14 & endoscopic & $12(86)$ & 3.7 \\
Skreden, 1987 [10] & 12 & endoscopic & $10(83.3)$ & 2 \\
Ambrosetti, 2008 [4] & 12 & endoscopic & $12(100)$ & 1.4 \\
Placer, 2010 [16] & 26 & endoscopic & $23(88.5)$ & 2.0 \\
Delaunay-Tardy, 2003 [13] & 27 & endoscopic & $41(77.1)$ & 2.0 \\
Pietropaolo, 1990 [12] & 42 & endoscopic vs. bougienage & $67 \%(63 / 94)$ & 2.5 \\
Suchan, 2003 [15] & 94 & endoscopic \& fluoroscopic & $71 \%$ complete & 1.0 \\
Di, 2005 [8] & 17 & fluoroscopic & $29 \%$ partial & \\
& & &
\end{tabular}

\section{Outcomes}

Dilatation of strictures was not associated with any mortality. Complications were noted in 12 patients $(2.9 \%)$ and in 850 procedures (1.4\%). These included intestinal perforations in $1.1 \%(9 / 850)$, abscesses in $0.2 \%$ $(2 / 650)$ and post-procedure pyrexia $0.1 \%(1 / 850)$. All patients who had bowel perforation needed operative intervention. One patient had a defunctioning stoma formation by repeated dilatation of an anastomotic stricture and subsequent successful stoma closure [9], 1 patient had a Hartmann's procedure and another had revision of anastomosis with covering stoma that was subsequently successfully reversed [7]. One study that described 5 perforations (5/94) and 2 abscesses did not specify the type of intervention for the complication [15]. Outcomes of dilatations were reportedly differently in the studies. These could be broadly divided into three categories

\section{Requiring Surgery}

A total of five studies reported failure as the requirement for revisional surgery. Solt et al. [11] stated that in their series 17 of 57 patients (30\%) needed reoperation. The reason was recurrence of disease in 2 patients, suspected recurrence in 6 , scarring stenosis not solved by dilatation in 8 , and pararectal cavity in 1 patient. The mean follow-up period in this study was 6.3 years (range 7 months to 17 years). The Hull series [7] described 24 of $33(72.7 \%)$ patients as not needing surgery with followup from last dilatation of median 18.5 months (range 0.59-87.6, IQR 4.39-37.1), and patency rates from time of first dilatation (irrespective of subsequent dilatations) at 1, 2 and 5 years as $69.8 \%$ (95\% CI 48.4-83.8\%), 69.8\%
(95\% CI 48.4-83.8\%) and 62.3\% (95\% CI 39.1-79.5\%). Of the 9 patients whose dilatation failed, 6 had a defunctioning stoma and 3 went to have revision of anastomosis. Placer et al. [16] described success in $88.5 \%$ (23/26) with a follow-up of 39 months (range 23-49 months). The 3 patients who had unsuccessful dilatation went on to have operative intervention and reanastomoses. Delaunay-Tardy et al. [13] noted success in $77.7 \%$ (21/27) patients with a follow-up range of 4 months to 8 years. Of those who underwent unsuccessful dilatation, 4 patients subsequently required revisional surgery and 2 had self-expanding metal stents inserted. Suchan et al. [15] confirmed success of stricture dilatation in $67 \%$ (63/94) patients. Of the 17 patients who did not benefit, 10 had benign restenosis, 5 suffered perforation during procedure (treatment not specified) and 2 developed abscesses. In addition, 7 patients were lost to follow-up and a further 7 developed local recurrence of cancer.

\section{Requiring Redilatation}

Three studies reported failure as need for dilatation again after a course of apparently successful dilatation(s). Araujo et al. [5] reported no need for redilatation in 18 of $22(81.8 \%)$ patients, with a follow-up of 2 years for each patient. The ultimate outcome of these redilatations was not described. Di et al. [8] reported that there was no necessity for redilatation in 16 of 17 (94\%) patients with an average follow-up period of 23 (range 1-62) months, and for that redilatation case to have satisfactory function at 7 months of follow-up. Venkatesh et al. [12] reported initial successful dilatation in 24 of 25 (96\%) of cases, with no stricture recurrence at yearly endoscopic review in the 
23 patients followed up, for 10, 7, 6 patients for $>3,>2,1$ years respectively.

\section{Relief of Symptoms}

Four of the studies reported relief of symptoms as a measure of success. Di et al. [8] reported 'complete' symptomatic response in 12 of $17(71 \%)$ and incomplete in the remainder, despite 'good' flow of contrast media through the dilated stricture in all. Johansson [9] reported that of the 14 patients followed up, 2 (14\%) had a 'poor' functional result (symptom of diarrhoea unchanged despite satisfactory dilatation) with a mean follow-up of 16.3 (range 3-54) months. Skreden et al. [10] reported that symptomatic relief was obtained in 10 of 11 patients (90.9\%) with 10 (SD 7, range 1-24) months of followup, and the remaining patient 'had diarrhoea until death from cardiac failure a year later'. Pietropaolo et al. [14] reported a failure rate of $2.4 \%(1 / 42)$ in their series. They also concluded that success of a single session of endoscopic dilatation $(76.9 \%)$ was significantly higher than bougienage (51.8\%).

One article had unclear outcomes. Ambrosetti's [4] cohort of 68 laparoscopic sigmoid colectomies had 12 patients (17.6\%) who underwent anastomotic stricture dilatation. The study described successful dilatation in all cases with no complications. However, there was no description of what a 'successful' outcome was, and the follow-up period post-dilatation was not stated.

One article reported on duration of response to a course of dilatation(s) before another was needed, comparing fluoroscopic and endoscopic methods. Di Giorgio et al. [6] reported that with a follow-up of either 24 months (maximal planned follow-up) or that when restricturing occurred, 'duration of response to dilation' was mean 294.2 days (SD 149.3) $(\mathrm{n}=15)$ by the 'throughthe-endoscope' technique versus mean 560.8 days (SD 248.5) ( $\mathrm{n}=15)$ for the 'over-the-wire' technique. No statement was given as to the number of patients restricturing, nor of patency rates over time, or of the outcome of treatments given upon restricturing.

\section{Risk Factors for Dilatation Failure}

Only three papers assessed for risk factors for dilatation failure.

The Hull series [7] reported that the chance of needing further stricture intervention (dilatation or surgery) was similar for a first or second dilation, but significantly worse in those having a third or subsequent dilatation (HR 1.0, HR 0.94 (95\% CI 0.47-1.89)) and HR 3.42 (95\% CI 1.51-7.71) respectively. Indeed, all 9 of these third or subsequent dilatations, performed in 5 patients, were futile in leading either to a further dilatation or ultimately surgery $(9 / 9,100 \%$ (95\% CI 66.3-100\%)), as compared to 22 of 41 first or second dilatations in 23 patients requiring either further dilatation(s) or surgery $(53.7 \%$ (95\% CI 37.4-69.3\%)). Araujo et al. [5] reported a non-significant trend between number of dilatations in an initial dilatation course and subsequent restricturing in a 2-year follow-up period for each patient. For patients having 1, 2,3 or 4 dilatations initially, restricturing subsequently occurred in $1 / 6,0 / 8,2 / 7$ and $1 / 1(p=0.08)$. These patients were reported to have then undergone further dilatations, but the ultimate outcome of these (e.g. need for surgery or otherwise) was not reported. Suchan et al. [15] and Delauny-Tardy et al. [13] showed that stricture dilatation was more successful in patients whose primary operation was for benign disease. Successful dilatation was seen in 59 and $61.5 \%$ in cancer resections and 88 and $92.8 \%$ in benign resections respectively. No association was seen between a dilatation failure requiring surgery, a history of anastomotic leak $(p=0.36)[7,15]$, a history of irradiation $(p=0.095)[7,15]$ and rapidity of stricture symptom onset after the anastomotic formation date $(\mathrm{p}=0.41)[7]$.

\section{Discussion}

The incidence of anastomotic stricture is difficult to discern. The Cochrane collaboration meta-analysis of randomised control trials that compared stapled versus hand-sewn colorectal anastomosis [17] listed an incidence of colorectal anastomotic stricture rate of 5.0\%, and for studies specifying supra- or infraperitoneal level subcategories, a 2.8 and $9.8 \%$ incidence. A qualification on the one hand was '... the majority of the studies (7/9) considered this complication to be irrelevant from a clinical point of view, given the favourable outcome with conservative treatment in the great majority of the cases', but on the other hand, '... the length of follow-up for assessing stenosis varied a lot between the different studies, which made comparison excessively difficult and may have made the overall result imprecise. Actuarial calculations, instead of crude ones, may be more appropriate in this context'.

The clinical anastomotic dehiscence in this metaanalysis were 9.1, 3.3 and $9.3 \%$ respectively and for radiologic leak 4.7, 7.8 and $12.1 \%$ respectively. Presumably some leaks would be managed non-operatively possibly predisposing to stricture formation subsequently. 
The proportion of patients with rectal stricture selected for particular forms of treatment is also difficult to know, as to the best of our knowledge there is no paper describing a series of rectal strictures and the treatments allocated. Rather, most articles are series reporting on a particular treatment technique. Therefore, the nature of cases referred for balloon dilatation is difficult to ascertain. Compounding this is the lack of descriptions of actual width and lengths of strictures treated with balloon dilatation; the only two papers reporting this would suggest strictures have been relatively short (mean $14.3 \mathrm{~mm}$ ).

As mentioned in the results, it is possible that around $2.1-8.6 \%$ of all patients with a rectal anastomosis may present for dilatation, and that around 3 patients per year will be referred for treatment in medium- to large-sized centres. In this context, and further anecdotally, we feel that rectal anastomotic strictures are not uncommon, that a good proportion are managed by retaining existing faecal diversion (stoma) only, and that more will be referred for balloon dilatation than would be revisional surgery.

So, for a relatively common complication, with a significant impact on the patient's quality of life and with certain treatment options being quite formidable (e.g. redo surgery), there is relatively little data on the management of this complication, from selection for treatment type through to the results of that treatment.

\section{Conclusion}

This review would suggest that relatively short strictures have been chosen for balloon dilatation, and that the results have had a very low major morbidity (2.9\%) and mortality $(0 \%)$ rate. Around three-quarters of patients referred and selected for this treatment will be satisfactorily managed by dilatation alone. There is some evidence that dilating for the third or subsequent time may be futile. For a common enough condition, there are very few studies reported, all retrospective case series in nature, and these have reported on disparate outcome measures. There is little in the literature to guide selection of one therapy type over another for rectal anastomotic stricture.

\section{References}

1 Macrae HM, McLeod RS: Handsewn vs. stapled anastomoses in colon and rectal surgery: a meta-analysis. Dis Colon Rectum 1998;41: 180-189.

$\checkmark 2$ Luchtefeld MA, Milsom JW, Senagore A, Surrell JA, Mazier WP: Colorectal anastomotic stenosis. Results of a survey of the ASCRS membership. Dis Colon Rectum 1989;32: 733-736.

-3 Garcea G, Sutton CD, Lloyd TD, Jameson J, Scott A, Kelly MJ: Management of benign rectal strictures: a review of present therapeutic procedures. Dis Colon Rectum 2003;46: 1451-1460.

-4 Ambrosetti P, Francis K, De Peyer R, Frossard JL: Colorectal anastomotic stenosis after elective laparoscopic sigmoidectomy for diverticular disease: a prospective evaluation of 68 patients. Dis Colon Rectum 2008;51: 1345-1349.

5 Araujo SE, Costa AF: Efficacy and safety of endoscopic balloon dilation of benign anastomotic strictures after oncologic anterior rectal resection: report on 24 cases. Surg Laparosc Endosc Percutan Tech 2008;18:565-568.
6 Di Giorgio PF, De Luca L, Rivellini G, Sorrentino E, D’amore E, De Luca B: Endoscopic dilation of benign colorectal anastomotic stricture after low anterior resection: a prospective comparison study of two balloon types. Gastrointest Endosc 2004;60:347-350.

7 Hull Series: Case series from Hull. Unpublished data.

-8 Di ZH, Shin JH, Kim JH, Song H-Y: Colorectal anastomotic strictures: treatment by fluoroscopic double balloon dilation. J Vasc Interv Radiol 2005;16:75-80.

-9 Johansson C: Endoscopic dilation of rectal strictures: a prospective study of 18 cases. Dis Colon Rectum 1996;39:423-428.

10 Skreden K, Wiig JN, Myrvold HE: Balloon dilation of rectal strictures. Acta Chir Scand 1987;153:615-617.

11 Solt J, Hertelendy A, Szilagyi K: Long-term results of balloon catheter dilation of lower gastrointestinal tract stenoses. Dis Colon Rectum 2004;47:1499-1505.

-12 Venkatesh KS, Ramanujam PS, McGee S:Hydrostatic balloon dilatation of benign colonic anastomotic strictures. Dis Colon Rectum 1992;35:789-791.
13 Delaunay-Tardy K, Barthélémy C, Dumas O, Balique JG, Audigier JC: Endoscopic therapy of benign colonic post-operative strictures: report on 27 cases. Gastroenterol Clin Biol 2003;27:610-613.

14 Pietropaolo V, Masoni L, Ferrara M, Montori A: Endoscopic dilation of colonic postoperative strictures. Surg Endosc 1990;4: 26-30.

15 Suchan KL, Muldner A, Manegold BC: Endoscopic treatment of postoperative colorectal anastomotic strictures. Surg Endosc 2003;17: $1110-1113$

16 Placer C, Urdapilleta G, Markinez I, Mugika F, Múgica JA, Elósegui JL, Murgoitio J, Irazusta M, Enríquez-Navascués JM: Benign anastomotic strictures after oncologic rectal cancer surgery. Results of treatment with hydrostatic dilation (in Spanish). Cir Esp 2010; 87:239-243.

17 Lustosa SA, Matos D, Atallah AN, Castro AA: Stapled versus handsewn methods for colorectal anastomosis surgery. Cochrane Database Syst Rev 2001;3:CD003144. 